\title{
Max Planck School of Photonics: research-oriented photonics education in a network of excellence throughout Germany
}

Reinhard Geiss, Julia Hengster, Thomas Kaiser, Gerd Leuchs, Andreas Tünnermann

Reinhard Geiss, Julia Hengster, Thomas Kaiser, Gerd Leuchs, Andreas Tünnermann, "Max Planck School of Photonics: research-oriented photonics education in a network of excellence throughout Germany," Proc. SPIE 11143, Fifteenth Conference on Education and Training in Optics and Photonics: ETOP 2019, 1114303 (2 July 2019); doi: 10.1117/12.2524606 Photonics: ETOP 2019, 2019, Quebec City, Quebec, Canada 


\title{
Max Planck School of Photonics: research-oriented photonics education in a network of excellence throughout Germany
}

\author{
Reinhard Geiss ${ }^{\mathrm{a}, \mathrm{b}^{*}}$, Julia Hengster ${ }^{\mathrm{a}, \mathrm{c}}$, Thomas Kaiser ${ }^{\mathrm{b}}$, Gerd Leuchs ${ }^{\mathrm{a}, \mathrm{d}}$, and \\ Andreas Tünnermann ${ }^{\mathrm{a}, \mathrm{b}, \mathrm{c}}$ \\ ${ }^{a}$ Max Planck School of Photonics, Albert-Einstein-Str. 7, 07745 Jena, Germany \\ ${ }^{\mathrm{b}}$ Abbe Center of Photonics and Abbe School of Photonics, Friedrich Schiller University Jena, \\ Albert-Einstein-Str. 6, 07745 Jena, Germany \\ ${ }^{\mathrm{c}}$ Fraunhofer Institute of Applied Optics and Precision Engineering, Albert-Einstein-Str. 7, 07745 \\ Jena, Germany \\ ${ }^{\mathrm{d}}$ Max Planck Institute for the Science of Light, Staudtstraße 2, 91058 Erlangen, Germany \\ * reinhard.geiss@uni-jena.de, Phone +49 3641 9-47965,www.photonics.school
}

\begin{abstract}
The Max Planck School of Photonics (MPSP) provides and coordinates an integrated program for the photonics education of graduate and doctoral students in a network of excellence of German universities and research institutions. Students can choose to start the program with a qualification phase to obtain a photonics Master's degree from one of three teaching universities in Erlangen, Jena or Karlsruhe. The subsequent $\mathrm{PhD}$-phase lasts three years and the research work is conducted under the supervision of one out of 45 Fellows of the MPSP. Students already holding a qualifying Master's degree can apply to directly enter the $\mathrm{PhD}$-phase of the program. Apart from an excellent network of renowned scientists and research institutions, the MPSP offers generous financial support and a supporting curriculum to foster interactions between the different photonics disciplines, to strengthen the research network and to advance personal and professional skills. Maintaining and supporting active interactions of the MPSP research network at all levels is challenging and will be backed up by employing digital learning tools and platforms. Here, we will present our recent findings on chances and opportunities provided by the MPSP's educative approach.
\end{abstract}

Keywords: graduate school, digital learning, Max Planck School of Photonics, Max Planck Schools

\section{MAX PLANCK SCHOOLS}

In recent years, a vast variety of technologies contributed to advancing our lives in regards of health, social communication and in the form of an overall preferable lifestyle. While scientists are still exploring the boundaries of said technologies, some of them remain unused. To use these chances and to further advance scientifically and technologically, Max Planck Schools were founded by Germany's most notable institutions, such as the Max Planck Society for the Advancement of Science (MPG) and universities all over Germany with support from the German Federal Ministry of Research and Technology.

\subsection{Mission}

Based on the idea of Synergy and with a holistic approach, MPS aim to capitalize on the already existing multitude of high-class German scientists and teaching structures to create a "whole that is greater than the sum of its parts". Therefore, they connect excellent scientist not only from Germany, but also from all over the world to help them advancing their own studies by including ideas and findings that are still to manifest themselves in the scientific discourse. Thus understanding themselves as a hub for advanced scientific communication. 
However, the ideas of MPS do not stop there. Keeping the future of science and the ongoing need for excellent researchers in mind, MPS also strive to support and encourage young scientist still to come. Students receive a non-refundable scholarship as well as the access to different courses and workshops both aiming for scientific and personal growth. Selected Fellows aid their students through this progress, supporting them to form further knowledge and new ways of thinking.

Lastly, MPS attempt to enhance the worldwide visibility of German universities and research institutions. While already being a country with a terrific amount of brilliant minds and high standards, German universities are still to reach the prestige to be named with world-class universities such as the famous Ivy League schools. But not only universities can benefit from the MPS being an acclaimed institution, scientists being part of the MPS alumni program can refer to their high standard education with the MPS.

\subsection{The variety of science}

While differentiation in education as well as in science is becoming increasingly relevant recently, the MPS attempt to find new ways to connect people from different research fields and their theories with each other. Starting with three different schools, MPS are going to be an umbrella term for high-class education and research for a variety of different science fields. The three MPS already existing are the "Max Planck School of Cognition", the "Max Planck School Matter to Life", and the "Max Planck School of Photonics" all based on different concepts and institutions throughout Germany. The focus of this article is going to be on the "Max Planck School of Photonics" (MPSP). However, to get a deeper understanding of MPS' focus the two other Schools will be introduced briefly in the following. Students interested in molecular dynamics, super-resolution microscopy or bioengineering, and anything surrounding these matters can deepen their understanding and advance their studies as part of the "Max Planck School Matter to Life". Three universities act as "teaching universities" for their respective studies and connect their findings to figure out unanswered questions regarding life and lifelike materials. Heidelberg University focusses on studies in "Molecular Systems Chemistry and Engineering", while students attached to "Complex Systems and Biological Physics" can research at the Göttingen University. Adding a third string of science to this field, the Technical University of Munich offers an education in "Bioengineering". But the MPS include not only natural science such as biology, physics and chemistry. As part of the "Max Planck School of Cognition" students can connect science and humanities such as psychology or philosophy. Here, students can decide between a large number of institutions all over Germany. Universities included in their program are, for example, the Ludwig Maximilian University Munich, being one of the most respectable universities in Germany.

\subsection{Structure}

To support autonomous research and active scientists MPS are aimed to be constructed bottom-up. While guided by the MPG headquarters, the MPS act mostly independent. Rules of procedures, a cooperation agreement as well as general board meetings secure the high standards of all associated universities and institutions. A management board headed by the MPS speakers directs the main concerns of the program and proposes new members and locations. On School level, the organization is different for each school. For the MPSP working groups govern the implementation and realization of the school's program and actions. The MPSP advisory board is composed of representatives from science, academia and industry. A coordination office supports the board and working group activities, organizes and implements curricular activities and keeps contact to the students.

\section{MAX PLANCK SCHOOL OF PHOTONICS}

Besides the "Max Planck School Matter to Life" and the "Max Planck School of Cognition", the "Max Planck School of Photonics" (MPSP) is mainly focusing on physical phenomena in the field of optics and photonics.

\subsection{Aspiration}

The primary goal of the MPSP is obtaining a more complex and better overall understanding of light. To reach this goal, MPSP is creating a network of excellence, consisting of already established scientists in the field of photonics as well as young and dedicated students with an academic background in physics. But not only does this network of excellence create a bridge between knowledge based on long-standing work in the field of photonics and fresh ideas of uncorrupted minds, it also aims to connect different scientific disciplines, facilities and people all over the world. MPSP therefore operates with a variety of methods, some of them already established at universities, some of them being an adaption to fast-paced progress in technology. 
Students can learn about new scientific findings or solidify common concepts through interdisciplinary symposiums, digital lectures or seminars, tutorials and practical courses. To foster everyone's needs, students can choose their own activities and can find their own learning styles, as individuality is one of MPSP strengths. The MPSP adapts its teaching portfolio to the different needs and existing knowledge of participating students. For students it is possible to visit preparatory courses and lectures designed for beginners and advanced students as well as specialization lectures. As MPSP is aware of the fact, that excellent scientists are not only vessels of knowledge, they encourage their students to take part in events to improve their networking and soft skills.

\subsection{Benefits for students}

The MPSP is supporting its students in a many different ways. The MPSP offers the students to explore their own ways of learning to reach their full potential. Thus, making the learning process as individual as possible. Getting students access to high quality laboratories and allowing them to interact with established and excellent researchers from all over Germany on a very personal level (due to a co-supervision concept, networking events) further their superiority compared to classical $\mathrm{PhD}$ studies. Additionally, a strong connection to the private sector is supported within the MPSP program providing an excellent base for a future career in both academia and industry.

The MPSP is aiming to attract dedicated and excellent student from all over the world. It is constantly searching for bright minds to advance the MPSP and universities visibility in the world and to establish itself as a high-class institution. Bringing those two objectives together, means creating a learning environment where students and Fellows can both advance, pushing each other to new heights.

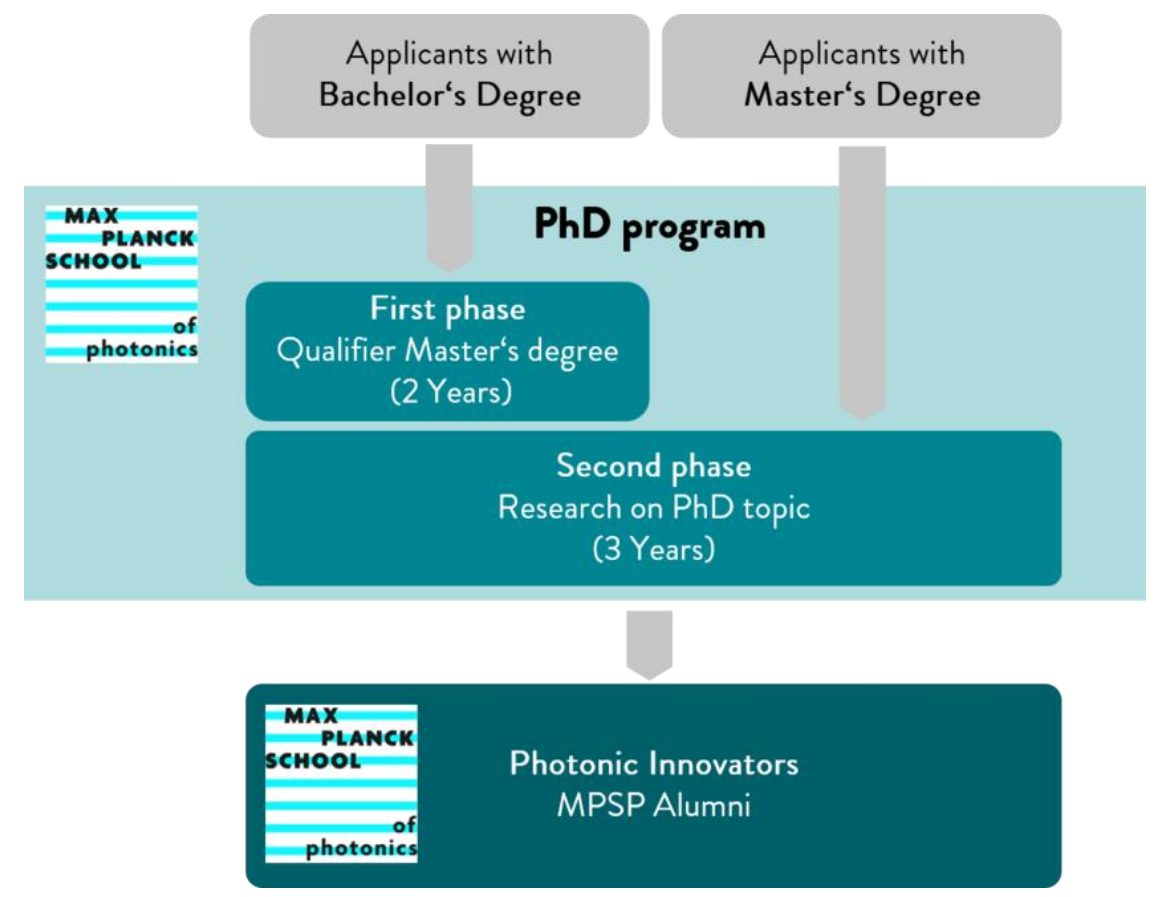

Figure 1: The Max Planck School of Photonics offers a two-phase PhD program to excellent students from all over the world. In the first phase, successful applicants pursuing or holding a Bachelor's degree obtain a Master's degree from one of three teaching universities in Erlangen, Jena, or Karlsruhe. The first phase has a duration of two years and, if completed successfully, directly qualifies for entering the second phase. The second phase is the research work on a PhD project. The PhD project is conducted in a group of one of the MPSP-Fellows under the supervision of this Fellow. The MPSP-Fellows are distributed amongst seven locations in Germany. Students can apply to any of the Fellows' research topics. The second phase of the program has a planned duration of three years.

Most noticeable and unusual for Germanys education system is that the MPSP PhD program accepts students already after completion of their Bachelor's degree. Students holding or expecting one of the following degrees are eligible to apply at 
the MPSP: Bachelor/Master of Science (B.Sc./M.Sc.), Bachelor/Master of Engineering (B.Eng./M.Eng.) or Bachelor/Master of Technology (B.Tech./M.Tech) with significant exposure to physics, engineering or a related subject. Those students enter the MPSP PhD program's first phase, which includes participation in the photonics Master's degree study program at one of the three teaching in either Jena, Karlsruhe or Erlangen.

The way towards the photonics Master's degree is characterized by the flexibility of the individual degree programs and a constant positive, competitive learning environment with all courses taught in English language. Students admitted to the MPSP receive a generous non-repayable scholarship, which allows them to fully concentrate on their studies without having to invest time in raising additional funding. Moreover, this stipend enables flexible mobility within the research network throughout the first phase of the program.

In the second phase, the students enter their PhD projects supervised by the MPSP's Fellows. As part of the MPSP, the Fellows are invested in the advancement of Germany's photonics research and, therefore, interested in pushing their students to the fullest potential. All of the participating institutions are providing laboratories with equipment complying with the high requirements set by the MPSP.

Students benefit from a strong network the MPSP is creating for their members. Being part of this network of excellence improves career opportunities after graduation. Not only due to the top-class education obtained through the MPSP Fellows, but also due to the connections and prestige received by being part of the network. Moreover, the career path into the private sector is addressed already at an early stage of education. In this way, the MPSP participants are ideally prepared for careers in both academia and industry. By participating in courses of the MPSP curriculum that target to achieve better soft skills students can improve their chances to enter a successful career in world-leading photonics companies.

\section{ADVANTAGES AND POSSIBILITIES OF PHOTONICS}

Of course, one of the main reasons to becoming part of the MPSP for young scientist should be the interest and the passion for everything concerning the matter of photonics.

\subsection{What photonics can do}

There is no doubt that photonics is one of the most important and, due to its many possibilities still to explore, also one of the most interesting branches of physics and technology. Apart from being one of the newer research branches coming into being in modern science, photonics already came a long way. While being mainly associated with science photonics is also a big part of modern life and culture. As in everyday life, modern industries are impossible to imagine without the photonic technologies as it is used in production and quality management. Human-machine interaction as part of the digital revolution is based on photonic devices. Photonics provides solutions to the ongoing struggles concerning humans use of energy. Already it is a dynamic branch of economy and a driving force behind countless innovations and possibilities.

It even proves to be a more than necessary part of other scientific disciplines working as a catalyst as well as contributing to the ever-growing progress in science. On a microscopic scale, researches seek for answers observing single molecules, while, on the other hand, the greatest cosmic events are observed using optical apparatuses, making photonics an intrinsically interdisciplinary. Its importance for modern sciences can be shown by its competitive success regarding the Nobel prices. Whilst some nobel prices are directly related to photonic research, many more utilize optics and photonics.

\subsection{Photonics in Germany}

Germany as an innovation seeking nation is already exploring the possibilities and boundaries of photonics. Looking at scientific publications released in the field of photonics, the quantity of German publications in this field are pushing it to the third place. Which is no surprise seeing how important photonic innovations are for the German industry.

All of this relies on highly qualified specialists, which are greatly demanded by the photonics industry in Germany. At the same time, german industries and research are facing shorter cycles of innovation, the ongoing exhaustion of physical and technical resources and the broad technology chains. To keep its status as one of the worlds leading photonic nations, Germany is in need of a way to expand their search for new highly qualified scientist and researcher to a worldwide range. 


\section{THE EXCELLENCE OF MPSP AND ITS ASSOCIATED PARTNERS}

Diving deeper into the matters and the structure of the MPSP one can wonder what exactly the claim of excellence in a MPSP's network of excellence is based on. Besides a well thought of structure and organization the main key to MPSP's excellence are the people and institutions that constitutes it.

\subsection{Universities and research institutions}

It is how the MPSP network is created and how it connects junior and senior researchers with each other making it a network of excellence. It is enabling communication without spatial and institutional boarders, the organization and how it is promoted is a considerable part of the success. All of the universities and research institutions building the MPSP are able to look back at a long history of supporting future scientist and providing their respective communities with amazing researches and discoveries. The universities and research institutions associated with the MPSP are meeting high standards and are distributed all over Germany.



Figure 2: The partners of the Max Planck School of Photonics are the University of Hamburg, the University of Göttingen, the RWTH Aachen University, the Friedrich Schiller University Jena, the Friedrich-Alexander University of Erlangen-Nuremberg, the Karlsruhe Institute of Technology, and the Ludwig Maximilian University of Munich. Together, they represent the german excellence in the field of photonics and contribute to research and education. Also part of the MPSP are several institutes from the four biggest German research societies: the Fraunhofer Institutes in Jena (Institute for Applied Optics and Precision Engineering) and Aachen (Institute for Laser Technology), the Max-Planck-Institutes in Erlangen (Institute for the Physics of Light) and Munich (Institute for Quantum Optics), the Deutsches Elektronen Synchrotron( (DESY) in Hamburg, the Helmholtz Centre for Heavy Ion Research in Jena and the Leibniz Institute for Photonic Technologies in Jena. These locations are not only epicenters in photonic research and origin of dynamic industry, but also internationally big players in academia and industry related teaching. The universities of Erlangen, Jena and Karlsruhe act as teaching universities with their excellent, accredited, well established and international master courses in the field of photonics.

The following nine research institutions are part of the MPSP: The "Fraunhofer Insitute for Applied Optics and Precision Engineering" (IOF) based in Jena is specialized in developing innovative optical systems for the control of light. The "Fraunhofer Institute for Laser Technology" (ILT) covers a wide range of areas such as the development of new laser beam sources and components, precise laser based metrology, testing technology and industrial laser processes and is based in Aachen. The "Max Planck Institute for the Science of Light" (MPL) is one of three Max Planck institutes and is located in Erlangen. The MPL research focuses on topics such as nonlinear optics, quantum optics, nanophotonics, photonic crystal 
fibres, optomechanics, quantum technologies, biophysics, and links between physics and medicine. The "Max Planck Institute of Quantum Optics" (MPQ) is located in Munich. It is exploring the interaction of light and matter under extreme conditions. The "Max Planck Institute for Biophysical Chemistry" (BPC) in Göttingen is focused on the molecular processes that drive and regulate the complex processes of life itself. Students can also push their research activities in one of the research centers of the Helmholtz-Associations, the "Deutsche Elektronen-Synchroton", also called DESY, being one of the world's leading synchrotron light sources. A similar topical focus has the "Helmholtz-Institute Jena" in Jena, as it is part of the "Helmholtzzentrum für Schwerionenforschung" (GSI). Finally yet importantly MPSP is supported by the "Leibniz Institute of Photonic Technology" (IPHT), which is investigating photonic and biophotonic processes and systems to address issues in the fields of medicine and the life and environmental sciences. All of these institutions are of most renowned institutions of the four major German research societies.

The same high standards are met by the universities that are hosting a larger number of the MPSP Fellows. Those universities can be found in Aachen, Erlangen, Göttingen, Jena, Karlsruhe and Munich, all of which are known for their future-oriented, yet established PHD-programs and many of them with a long history of terrific scientists studying, researching and teaching there. Taking a special role for the MPSP are the universities in Erlangen, Jena, and Karlsruhe as they are their designated teaching universities. All of them were chosen due to their significant role in the German photonics research as well as their reputation as top universities. They provide the MPSP with accredited, established, international photonics Master's degree courses.

Based in the heart of Thuringia students can find the "Friedrich-Schiller-Universität" (FSU) in Jena. The city is one of Germany's most beautiful student cities and also known as "Lichtstadt" which means "city of lights". Both, the city and the university have a long history with well-known scientists from a variety of different fields and are now location for researcher and scientists, who earned their worldwide recognition with their meaningful work. Here, students of the MPSP can learn everything there is to know about applied optics as well as laser physics.

A groundbreaking partnership was formed 2009 in Karlsruhe as the local university Universität Karlsruhe (TH) and the "Kernforschungszentrum Karlsuhe GmbH" (KfK) fused to the "Karlsruher Instiut of Technology" (KIT). Therefore, the KIT is also known as "the Research University in the Helmholtz Association". Besides contemplating the marvelous city, students can work on their career as graduates from KIT and are welcome workers for many companies. Furthermore, KIT can impress with a vast variety of research programs reaching from astroparticle physics to renewable energies. For MPSP students the most interesting part of the KIT is probably their focus on optoelectronics and solid-state optics.

Also able to impress with a long history, there is the "Friedrich-Alexander Universität Erlangen-Nürnberg" (FAU) located mainly in Erlangen. The FAU is one of the bigger universities in Germany and located in a comparable "small" city. In 2018, the FAU was named the most innovative university in Germany by the Reuters ranking. The FAU provides its knowledge about quantum optics and artificially structured materials to the MPSP.

\subsection{Fellows}

To earn its name as a "network of excellence" many excellent researchers joined the MPSP as Fellows. With their help, the MPSP can reach its goal to form a network of acclaimed researches from all over the world and to create a positive learning environment, open to students from different fields as well as from different countries.

All of the Fellows that are part of the MPSP already proved that they are capable and reliable scientists making an impact on the scientific world with their outstanding work. They all are eminent figures in the world of science.

\subsection{A network of excellence - choosing the right students}

The main concern of MPSP are of course the students taking part in the PhD-program and being the future of the photonic research and industry. They are according to MPSP the most important carrier of excellence and the power of innovation. Therefore, they are chosen carefully and based on a variety of different criteria including their performance in a specific entrance examination. Thanks to participation of already established study programs, the MPSP can rely on their local and international Master's degree programs "Master Program in Advanced Optical Technologies"(MAOT) in Erlangen, the "Karlsruhe School of Optics and Photonics" and the "Abbe School of Photonics" in Jena.

Students applying to the MPSP are required to go through a complex selection process. To meet the high standards of the MPSP they have to provide overall excellence. Students have to proof their personal suitability, academic education and research excellence. After applying online, they have to go through a multistep selection procedure taking into consideration a motivation letter, curriculum vitae, letters of recommendation, personal research objectives and language 
skills. Interviews, tests and matching events are ensuring that the MPSP is including only those students in the program that represent the aspirations and philosophy of the MPSP in the best way possible. Each cohort of the first phase is composed of approximately 20 students maximum per class, resulting in 40 students within the program's the first phase. The second phase is built from 20 transition students from the first phase and additional 20 Master's degree graduates from

other study programs. Keeping the program at such a small scale allows the MPSP to concentrate on the most promising students and offer them the best education possible.

\section{MPSP AS INTERDISCIPLINARY LEARNING ENVIRONMENT}

The MPSP is able to provide students a learning environment not to be found anywhere else. Due to the participation of the most renowned Fellows MPSP is able to provide students both excellent scientific quality and excellent scientific education. Bringing the modern advances of technologies into play, students can be part of all these aspects resulting in yet overseen connections and hopefully unparalleled discoveries.

One of the key components are going to be digital teaching courses. Proven to increase the effectiveness of learning, digital teaching is one of the newest, but yet still mainly unused learning platforms. As the majority of the ongoing students are going to be digital natives, born after 1990, this is reflecting their way of understanding and communicating. Besides taking part at classical forms of learning such as lectures and tutorials, students are engaging in the knowledge of excellent minds from all over the world and are also able to share their findings and thoughts with similarly minded students. The MPSP is more than excited to be a platform for all its students, researchers and scientists. The MPSP engages programs and methods of interaction to unite its members at universities and non-university institutions in an overarching network, bridging the gap between different location, institutions and working fields. 